\title{
Percepciones de Estudiantes Universitarios sobre una Evaluación Formativa en el Trabajo en Equipo
}

\section{University Students' Perceptions of a Formative Assessment in the Teamwork}

\author{
Sonia Asún Dieste * \\ Marta Rapún López \\ Ma Rosario Romero Martín \\ Universidad de Zaragoza, España
}

\begin{abstract}
El trabajo en equipo es una competencia muy valorada en el contexto laboral. No obstante, se reconoce como deficitaria en la formación inicial universitaria. Este artículo muestra las percepciones de los estudiantes universitarios sobre una evaluación formativa y compartida de la competencia de trabajo en equipo, implementada durante los cursos 2016-2017 y 2017-2018, en el tercer curso de Grado en Ciencias de la Actividad Física y del Deporte de la Facultad de Ciencias de la Salud y del Deporte de la Universidad de Zaragoza. El objetivo fue mejorar la competencia del trabajo en equipo y explorar las percepciones de los estudiantes. Tras la implementación de la experiencia se realizó un estudio cualitativo en el que se preguntó a 120 estudiantes mediante entrevistas semiestructuradas. Los resultados mostraron la eficacia de la evaluación formativa y compartida para elevar los niveles de implicación durante el trabajo en equipo, mejorar el desarrollo de la competencia y reconocer sensaciones de justicia en la calificación; si bien, también se evidenció la complejidad inherente a las relaciones interpersonales durante los trabajos en equipo, la necesidad de mayor apoyo docente ante los conflictos de grupo, la generación de clima negativo para el aprendizaje en determinados perfiles de grupos y alguna incoherencia sobre las negociaciones de la calificación.
\end{abstract}

Palabras clave: Educación superior; Aprendizaje activo; Evaluación formativa; Evaluación del estudiante; Retroalimentación; Cooperación educacional.

Teamwork is one of the most highly valued skills in future professionals who will be incorporated into the employment context. However, it is recognized how deficient it is in Higher Education. This article shows the students' perceptions about a formative and shared assessment of the teamwork competence produced during the 2016-2017 and 2017-2018 years in the Faculty of Health and Sports Sciences at the University of Zaragoza. The aim was to improve the teamwork and explore students' perceptions. After implementing a formative assessment we carried out a qualitative study in which we asked 120 students by semi-structured interviews. The results showed the effectiveness of formative and shared assessment to raise the levels of responsibility, the development of the teamwork and feeling of justice in the marks; but, it was also suggested the complexity inherent at interpersonal relationship skills during the team work, the need for greater teaching support in group conflicts, the creation of a negative climate for learning in particular group profiles and some inconsistencies in the negotiation of the marks.

Keywords: Higher education; Active learning; Formative assessment; Student evaluation; Feed-back; Educational cooperation.

*Contacto: sonasun@unizar.es

issn: 1989-0397

www.rinace.net/riee/

https://revistas.uam.es/riee
Recibido: $\quad$ 04 de diciembre de 2018

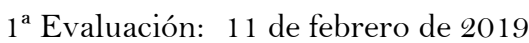

$2^{\text {a }}$ Evaluación: 13 de marzo d 2019

Aceptado: $\quad 15$ de marzo de 2019 


\section{Introducción}

El desarrollo de competencias en los estudiantes de Educación Superior está siendo una cuestión prioritaria de estudio en todas las universidades con sistemas de calidad en proceso. En este sentido se ambiciona también la calidad de la competencia de los estudiantes en el trabajo en equipo, así como, de la valoración que los profesores realizan de la misma. No resulta extraño encontrar, entre los diseños de asignaturas, actividades de trabajo en grupo que, normalmente, los profesores someten a evaluaciones finalistas. Sin embargo, es menos frecuente encontrar literatura versada en cómo acometen la evaluación del desarrollo de la competencia del trabajo en equipo los profesores de universidad que priorizan una evaluación formativa con propósitos de aprendizaje y no, únicamente, de calificación. En este estudio se presentan los resultados de la aplicación de una evaluación formativa del trabajo en equipo, que fue implementada durante dos cursos académicos, que priorizó el aprendizaje y que aportó datos sobre las percepciones de los estudiantes.

\section{Revisión de literatura}

\subsection{Las competencias en la formación universitaria}

Como se pone de manifiesto en la numerosa bibliografía actual sobre Educación Superior, la implantación del sistema ECTS no solo ha supuesto una transformación de la estructura organizativa universitaria, sino también, de los diseños curriculares y su implementación. Entre las distintas declaraciones institucionales a propósito de la convergencia europea sobre Educación Superior, la de Lisboa (Parlamento Europeo, 2000), incide en la necesidad de una formación integral para el individuo, más allá de la mera formación profesional en una determinada disciplina. Como consecuencia de ello, en los estudios e informes que se derivaron para dotar de consistencia teórica al reto que se le planteaba a los países, como fue el informe Tuning (González y Wagenaar, 2003), queda patente el compromiso de considerar los títulos universitarios en términos de resultados de aprendizaje y, particularmente, en términos de competencias, siendo ambos los que constituyen el núcleo central de la programación docente universitaria en la actualidad. A partir de esta concepción las competencias para la formación universitaria se organizan en dos grandes bloques: (1) competencias específicas de cada área temática, definidas como destrezas y conocimientos relativos a la disciplina concreta; (2) competencias genéricas o transversales, caracterizadas por ser comunes a cualquier titulación, como la capacidad de aprender, de tomar decisiones, de diseñar proyectos, etc., y estrechamente ligadas a las actuales demandas sociales y laborales (Michavila, Martínez, Martín-González, García-Peñalvo y Cruz-Benito, 2016). A su vez, las competencias genéricas se diferencian en: (1) instrumentales (habilidades cognoscitivas y capacidades metodológicas de adaptación al entorno, destrezas tecnológicas y lingüísticas); (2) interpersonales (capacidades individuales relativas a la capacidad de expresar los propios sentimientos, habilidades críticas y de autocrítica); y (3) sistémicas (destrezas y habilidades que conciernen a los sistemas como totalidad).

A pesar del escaso desarrollo de estudios relacionados con las competencias transversales y su evaluación, se han publicado algunos trabajos como el de De Miguel (2005), en el que las modalidades de enseñanza se desglosan en relación con el desarrollo de competencias y su evaluación; igualmente, el estudio de Villa y Poblete (2007), en el que se establecen 
niveles de competencias genéricas para facilitar el planteamiento de actividades de aprendizaje, así como, su evaluación; y más recientemente, se publican interesantes estudios que abordan este objeto de conocimiento (eg.: Clemente y Escribá, 2013; Martínez-Clares y González-Morga, 2018). No obstante, el ámbito de la innovación docente ha sido el más prolífero al respecto, produciendo y desarrollando proyectos (e.g. Pastor del Pino et al, 2017), a partir de grupos de profesores inquietos por la temática.

\subsection{La evaluación formativa como evaluación de competencias}

De los tipos de evaluación expuestos por Utha (2015), a saber: evaluación sumativa, evaluación continua sumativa o evaluación continua formativa, comienza a expandirse la idea de que si se desea avanzar en la evaluación de competencias no resulta útil una simple evaluación sumativa, o evaluación continua sumativa, pues ambas adolecen de la deseable potencia de la función formativa en la Universidad. Diversos autores coinciden en que la evaluación debería tener un carácter formativo y continuo (Brockbank y Mc-Gill, 2006; Denton, 2014; Ibarra y Rodríguez-Gómez, 2010); asímismo, ratifican que este tipo de evaluación consigue elevar las calificaciones de los estudiantes, en relación a otros itinerarios o modelos de evaluación menos formativos, lo que parece evidenciar un mayor efecto de aprendizaje (Lizandra, Valencia, Atienza y García, 2017). Igualmente, se considera que la evaluación llevada a cabo únicamente por el profesor, heteroevaluación, sería menos coherente con la filosofía de la programación por competencias que la coevaluación, autoevaluación u otras fórmulas de evaluación pactada, negociada y compartida con los estudiantes ( Bain, 2005; Boud y Falchikov, 2007; Brown y Glasner, 1999; López-Pastor, Pintor, Muros, y Webb, 2013). Se reconoce también que estas formas de evaluación presentan numerosas ventajas respecto de la implicación del alumnado y de su percepción sobre el aprendizaje (Carless, 2006; Hortigüela, Pérez-Pueyo y Abella, 2015). Romero, Fraile, López y Castejón (2014) obtuvieron en su estudio que a pesar de que para los alumnos la evaluación formativa suponía una mayor carga de trabajo, su satisfacción general era alta con el proceso y los aprendizajes conseguidos; a su vez, encontraron diferencias significativas en el rendimiento a favor de la evaluación formativa y continua en relación al examen final.

\subsection{La competencia de trabajar en equipo}

La competencia de trabajo en equipo, motivo en concreto del presente trabajo, se revela como un elemento importante, no solo como tal competencia, sino porque lleva consigo las capacidades de organización y gestión primordiales para los empleadores (SánchezElvira, López-González y Fernández-Sánchez, 2010).

En el proyecto Tuning esta competencia transversal se asocia a las habilidades de dirección, liderazgo y relación interpersonal. La competencia de trabajo en grupo se relaciona con todos los mecanismos que se ponen en marcha cuando se tiene que desarrollar una tarea, en la que se activan acciones de intercambio de ideas, interacción, colaboración y diálogo, de manera conjunta. También capacidades interpersonales como las que menciona Domingo (2008): "negociación, consenso, respeto, capacidad para comprender los puntos de vista de los demás, argumentar estructuradamente y de forma lógica y coherente los propios, expresarse con corrección, criticar sin herir, etc.,..." (p.232). 
Sánchez-Elvira et al. (2010), a partir de documentos de la UNED, desglosan la competencia de trabajo en grupo en habilidades para coordinarse con el trabajo de otros; negociar de forma eficaz; la mediación y resolución de conflictos; coordinar grupos de trabajo; de liderazgo.

Trabajar en grupo no consiste en la suma de tareas individuales enlazadas y con un formato final conjunto, si no en un proceso cuyo resultado es mucho más que la suma de las partes. Se necesita en opinión de Prieto, Alarcón y Fernández (2018) un objetivo común, relaciones interpersonales simétricas y reciprocas y la posibilidad de obtener una recompensa común en la resolución de la tarea. Efectivamente, se constata que en ocasiones el trabajo en grupo, más que una puesta en común y un aprendizaje colaborativo, negociado, coordinado, mediado..., es la suma de varias tareas que luego se funden en una producción única. Esto nos permite hacer una importante diferenciación entre el concepto de trabajar en grupo (varias personas tienen un objetivo que han de conseguir en conjunto), y trabajar en equipo (Domingo, 2008), donde el proceso de conseguir la tarea es en determinadas condiciones de interacción e implica todas las habilidades descritas, siendo esta última, la concepción con la que nos posicionamos.

Para Scallon (2004), el trabajo en equipo ha sido una de las competencias genéricas que los profesores universitarios han pretendido desarrollar con cierta asiduidad; pero, con frecuencia, no se ha tenido en cuenta el proceso y el aprendizaje de las habilidades necesarias de los estudiantes para conseguir una óptima cooperación. Como indicaba Zabalza (2012), "enseñar" una competencia y "emplear" las competencias son cosas distintas. Diversos autores (eg: Sánchez Elvira et al., 2010; Zabalza, 2012), han ayudado a operativizar la programación, desglosando las competencias en subcompetencias, organizándolas a modo de mallas; diseñando actividades de asimilación y evaluación; y analizando sus distintas lógicas internas para su secuenciación.

En cuanto a la investigación sobre la competencia de trabajo en equipo se ha enfocado en gran medida a cómo mejorar el resultado final de la tarea encomendada y a aspectos relacionados con las calificaciones, pues éstas reflejaban resultados individuales más que del grupo (Howe, 2011). Autores como Morales (2008) o Pérez-Pueyo, Hortigüela y Hernando (2013), han propuesto sistemas de reparto de notas para la calificación de los trabajos en equipo, en un intento de elevar la implicación y mejorar la justicia en la calificación; y otros, han defendido la idoneidad de que se integren valoraciones de profesores y estudiantes, mediante procesos de autoevaluación y co-evaluación (Proulx, 2009).

\subsection{Diseños de evaluación formativa para abordar la competencia de trabajo en equipo}

La implementación de los diseños docentes que se realizan con eł objeto de alcanzar eficacia en el desarrollo de la competencia de trabajo en equipo, requiere abordar con urgencia dimensiones funcionales, cognitivas y afectivas durante el proceso, en la misma medida que calificar los resultados de la producción teórica o práctica del equipo de estudiantes.

$\mathrm{Al}$ mismo tiempo, resulta imprescindible que en estos diseños se constituyan las bases para formular indicadores de nivel que puedan ser comprendidos y elaborados conjuntamente 
(González y Wagenaar, 2003). Y utilizar dichos indicadores en un proceso de evaluación continua formativa que se implemente durante el desarrollo del trabajo en equipo.

Por tanto, convendría que la programación de esta competencia se diseñase en los planes con indicadores de nivel consensuados, a nivel funcional, cognitivo y afectivo, y de fácil comprensión y aplicación por parte del equipo de profesores; resultaría óptimo igualmente, establecer gradaciones de la competencia en todos los cursos de los diferentes títulos de la formación universitaria, si bien, el desarrollo de esta competencia genérica deberá formar parte, irremediablemente, del aprendizaje a lo largo de la vida, tal y como propone la declaración de Lisboa (Parlamento Europeo, 2000).

En este estudio se intenta profundizar en las percepciones de estudiantes universitarios de tercer curso de Grado en Ciencias de la Actividad Física y del Deporte de la Universidad de Zaragoza sobre su aprendizaje de la competencia de trabajar en equipo tras la aplicación de una evaluación continua formativa y compartida. Durante la misma se realiza un diagnóstico inicial del perfil funcional, cognitivo y afectivo del equipo y se propicia la autorregulación de este perfil durante el proceso, mediante la autoevaluación y la evaluación entre iguales. Igualmente, se comparte y se dialoga sobre el sistema de calificación final del trabajo.

\section{Método}

\subsection{Enfoque metodológico}

Se realizó un estudio de diseño cualitativo (Creswell, 2011) basado en la fenomenología pues se intentó describir y entender el fenómeno desde las experiencias individuales y subjetivas de cada uno de los participantes expuestos al proceso de una evaluación continua formativa y compartida, tomando conciencia no solo de los fenómenos sino también del modo en que se presentaron y constituyeron los mismos (Aguirre-García y Jaramillo-Echeverri, 2012).

\subsection{Categorías de análisis}

Inicialmente se establecieron tres áreas de observación sobre las que se sustentaron las preguntas de las entrevistas semiestructuradas. La primera fue Percepciones y significados sobre el trabajo en equipo en la educación superior, la segunda fue Experiencias vivenciadas en la evaluación formativa del trabajo en equipo y la tercera, Percepciones sobre el sistema de calificación implementado en el trabajo en equipo. Sobre dichas áreas, que se obtuvieron a partir de la literatura y anteriores trabajos de campo (Strauss y Corbin, 2002) realizados por las autoras, surgieron las categorías y datos que se exponen en los resultados.

\subsection{Selección de participantes}

El estudio tuvo lugar en el tercer curso de Grado de Ciencias de la Actividad Física y el Deporte de la Universidad de Zaragoza, en el Campus de Huesca, concretamente en la asignatura de Diseño y Evaluación en la enseñanza de la actividad física y del deporte, durante los cursos académicos 2016-2017 y 2017-2018.

Participaron un total de 82 estudiantes de los 127 matriculados en la asignatura durante los dos cursos. Todos ellos se acogieron a la modalidad oficial de evaluación continua, y aceptaron participar en la evaluación continua formativa y compartida, comprometiéndose 
a involucrarse en una experiencia de buena práctica hasta el final del proceso, y responder a las entrevistas previstas. Los participantes fueron 62 hombres, y 20 mujeres con edades comprendidas entre los 20 y 32 años, siendo menores de 22 años el $83 \%$ de los alumnos y mayores de esa edad el $17 \%$.

El estudio se enmarcó en el seno de la Red Nacional de Evaluación Formativa y Compartida y ha sido reconocida por la Universidad de Zaragoza a través de los proyectos de innovación (PIDUZ_16_386) y (PIDUZ_17_396), siendo únicamente financiado en su segundo año de implementación y registro de datos.

\subsection{Instrumento}

Se utilizaron entrevistas semiestructuras que permitieron obtener flexibilidad en las respuestas. Durante el primer curso se realizaron de forma escrita y durante el segundo curso, se realizaron a través de un formulario Google Drive, intentando llegar a un mayor número de respuestas que recogiesen las experiencias, significado y compresión de los estudiantes en torno a la evaluación formativa en el trabajo en equipo (Patton, 1990), así como respuestas dirigidas a la temática concreta que experimentaron.

\subsection{Trabajo de campo}

Al inicio de la asignatura de cada uno de los cursos se explicó cómo se realizaría la evaluación continua y formativa de un proyecto, en el que los estudiantes debían trabajar en equipo cooperando, con el fin de defenderlo al final del proceso ante un tribunal de profesores y sus propios compañeros de clase. Se presentó el proyecto a los estudiantes, en el primer día de clase de cada uno de los cursos, y se les planteó la posibilidad de acogerse al sistema de evaluación continua formativa y compartida propuesto, así como, participar al final de curso en las entrevistas semiestructuradas para la recopilación de datos sobre sus percepciones.

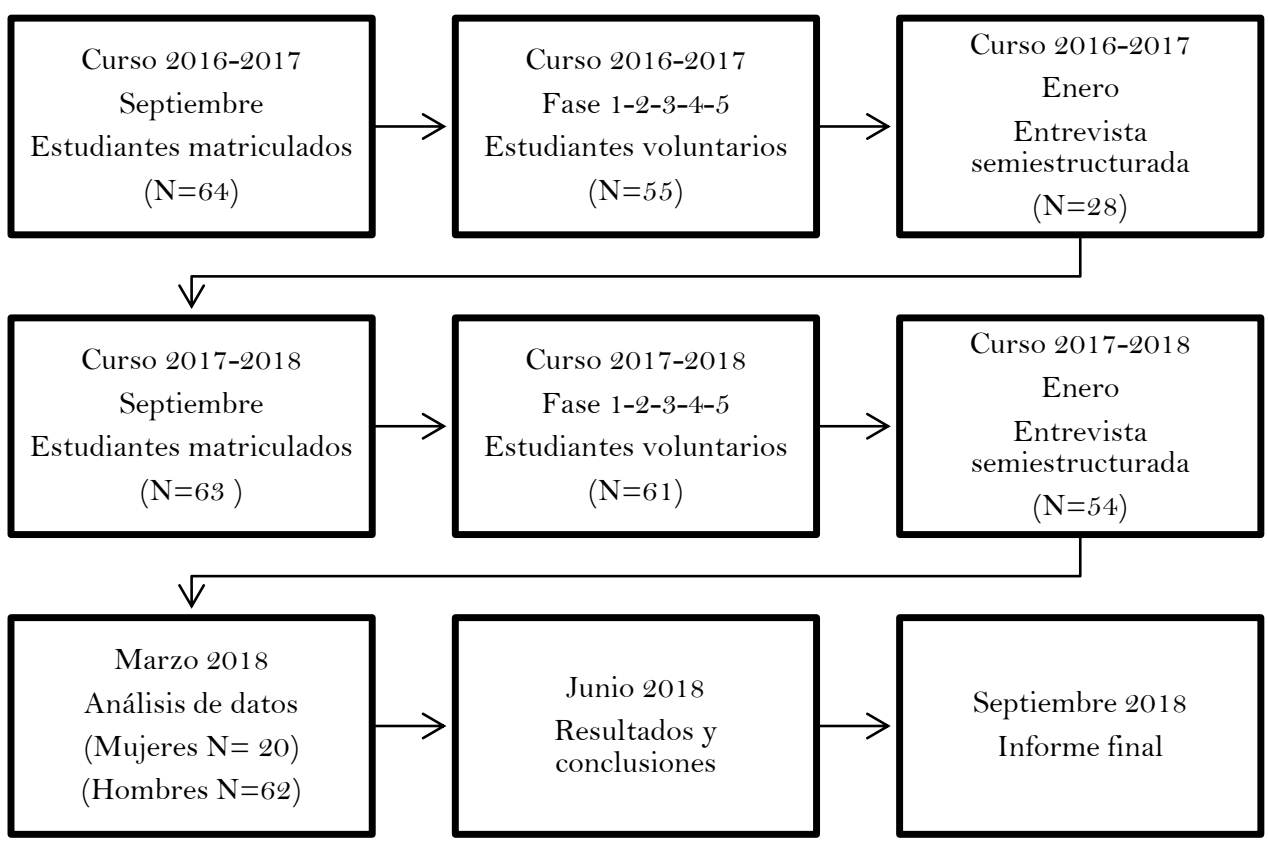

Figura 1. Esquema del estudio Fuente: Elaboración propia. 
Todos los asistentes aceptaron la propuesta $(\mathrm{N}=127)$, por lo que se realizaron actividades de evaluación continua formativa y compartida orientadas a la mejora y valoración del trabajo en equipo, durante cinco seminarios y dos tutorías, a los que asistieron los alumnos con su equipo de trabajo, que previamente habían elegido de forma libre, con la única condición de que fueran equipos de aproximadamente 14 personas. El proceso se estructuró en 5 fases que se repitieron en cada uno de los cursos (figura 1).

En la fase 1 se realizó, al inicio de la asignatura de cada uno de los cursos, una autoevaluación diagnóstica sobre el perfil del grupo en su nivel de competencia de trabajo en equipo, a partir de una adaptación de las aportaciones de Pastor del Pino et al (2017), para el establecimiento de niveles de competencia en el trabajo en equipo (figura 2).

\begin{tabular}{|c|c|c|c|c|}
\hline & Excelente & Admirable & Adquirido & $\begin{array}{l}\text { En vía de } \\
\text { adquisición }\end{array}$ \\
\hline $\begin{array}{l}\text { Acepta y cumple } \\
\text { las normas }\end{array}$ & $\begin{array}{l}\text { Propone normas y } \\
\text { vela por su } \\
\text { cumplimiento }\end{array}$ & $\begin{array}{l}\text { Participa en la } \\
\text { construcción de } \\
\text { normas }\end{array}$ & $\begin{array}{l}\text { Acepta } \\
\text { normas }\end{array}$ & $\begin{array}{lr}\text { Cuestiona } & \text { las } \\
\text { normas } & \text { para } \\
\text { adaptarlas a } & \text { sus } \\
\text { intereses y/o } & \text { no } \\
\text { acepta } & \text { las } \\
\text { normas } & \end{array}$ \\
\hline $\begin{array}{l}\text { Contribuye al } \\
\text { establecimiento y } \\
\text { aplicación de los } \\
\text { procesos del } \\
\text { trabajo en equipo }\end{array}$ & $\begin{array}{l}\text { Contribuye de } \\
\text { modo excelente al } \\
\text { establecimiento y } \\
\text { aplicación de los } \\
\text { procesos del } \\
\text { trabajo en equipo }\end{array}$ & $\begin{array}{l}\text { Contribuye de } \\
\text { modo adecuado } \\
\text { al establecimiento } \\
\text { y aplicación de los } \\
\text { procesos del } \\
\text { trabajo en equipo }\end{array}$ & $\begin{array}{l}\text { Contribuye poco } \\
\text { al establecimiento } \\
\text { y aplicación de los } \\
\text { procesos de } \\
\text { trabajo en equipo }\end{array}$ & $\begin{array}{l}\text { No contribuye al } \\
\text { establecimiento y } \\
\text { aplicación de los } \\
\text { procesos de } \\
\text { trabajo en equipo. }\end{array}$ \\
\hline $\begin{array}{l}\text { Actúa } \\
\text { constructivamente } \\
\text { para afrontar los } \\
\text { conflictos de } \\
\text { grupo }\end{array}$ & $\begin{array}{l}\text { Actúa de manera } \\
\text { excelente al } \\
\text { afrontar } \\
\text { constructivamente } \\
\text { los conflictos de } \\
\text { grupo }\end{array}$ & $\begin{array}{l}\text { Actúa de manera } \\
\text { adecuada } \\
\text { intentando } \\
\text { resolver } \\
\text { positivamente los } \\
\text { conflictos }\end{array}$ & $\begin{array}{l}\text { Actúa algo en los } \\
\text { conflictos pero la } \\
\text { crítica y el enfado } \\
\text { son más } \\
\text { frecuentes que el } \\
\text { intento de } \\
\text { resolver }\end{array}$ & $\begin{array}{l}\text { No actúa ante los } \\
\text { conflictos o lo } \\
\text { hace de modo } \\
\text { muy destructivo. }\end{array}$ \\
\hline $\begin{array}{l}\text { Con su forma de } \\
\text { comunicar } \\
\text { relacionarse } \\
\text { contribuye a la } \\
\text { cohesión de grupo }\end{array}$ & $\begin{array}{l}\text { Escucha y valora } \\
\text { las opiniones de } \\
\text { los demás } \\
\text { incitando a todos a } \\
\text { la participación }\end{array}$ & $\begin{array}{l}\text { Siempre escucha } \\
\text { y valora para } \\
\text { llegar a un } \\
\text { consenso entre } \\
\text { todos }\end{array}$ & $\begin{array}{lr}\text { Casi } & \text { siempre } \\
\text { escucha y valora } \\
\text { aunque a veces } \\
\begin{array}{l}\text { aporta } \\
\text { cuestiones }\end{array} \\
\begin{array}{l}\text { negativas } \\
\text { positivas }\end{array}\end{array}$ & $\begin{array}{lr}\begin{array}{lr}\text { Solo tiene } \\
\text { cuenta }\end{array} \\
\text { valoraciones que } \\
\text { están cerca de } \\
\text { sus intereses y/o } \\
\text { ni escucha ni } \\
\text { valora }\end{array}$ \\
\hline
\end{tabular}

Figura 2. Rúbrica elaborada para la autoevaluación en el trabajo en equipo Fuente: Adaptado de Pastor del Pino et al. (2017).

En la fase 2 se debatieron algunos elementos clave en el trabajo en equipo, como la definición de tareas y funciones, asignación de roles y normas, definición de objetivos, comunicación y responsabilidad. Además, se mostraron dos sistemas para calificar el trabajo en equipo mediante el reparto de la calificación para cada uno de los miembros del grupo a partir de lo planteado por Morales (2008): sistemas basados en el reparto de puntos en función de la contribución de cada miembro del equipo y sistemas basados en la evaluación mutua de todos los miembros del equipo de trabajo. Cada equipo se decantó por el sistema que consideró más útil y justo, diseñando todos los equipos sistemas basados en la evaluación mutua mediante rúbricas o escalas numéricas.

En la fase 3 (seminario 4) se realizó una autoevaluación y evaluación entre iguales mediante una escala de clasificación ordinal (tabla 2) para reconocer el estado del equipo hasta ese momento y dialogar sobre los posibles problemas o dificultades encontradas, buscando mejoras y regulando el proceso. 


\begin{tabular}{|c|c|c|c|c|c|c|c|c|c|c|c|c|}
\hline & \multicolumn{3}{|c|}{ Nombre alumno } & \multicolumn{3}{|c|}{ Compañero 1} & \multicolumn{3}{|c|}{ Compañero 2} & \multicolumn{3}{|c|}{ Compañero 3} \\
\hline & 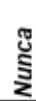 & 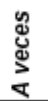 & 离 & $\frac{\mathbb{S}}{\mathbf{S}}$ & 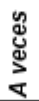 & 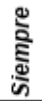 & 胥 & 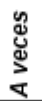 & 离 & 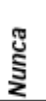 & 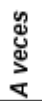 & 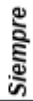 \\
\hline $\begin{array}{l}\text { 1.- He aceptado y cumplido las normas } \\
\text { de funcionamiento del grupo, } \\
\text { motivando, mostrado respeto y apoyo } \\
\text { hacia sus miembros. }\end{array}$ & & & & & & & & & & & & \\
\hline $\begin{array}{l}\text { 2.- He asistido y participado } \\
\text { activamente en las clases, reuniones y } \\
\text { debates internos del grupo. }\end{array}$ & & & & & & & & & & & & \\
\hline $\begin{array}{l}\text { 3.- He desempeñado el rol asignado a } \\
\text { partir de las competencias del trabajo } \\
\text { en equipo }\end{array}$ & & & & & & & & & & & & \\
\hline $\begin{array}{l}\text { 4.- He realizado positivamente mi parte } \\
\text { del trabajo y en los plazos acordados } \\
\text { por el grupo. (teniendo en cuenta que } \\
\text { su labor y actitud condicionan la de los } \\
\text { demás y el resultado final) }\end{array}$ & & & & & & & & & & & & \\
\hline $\begin{array}{l}\text { 5.- He identificado las pautas de } \\
\text { funcionamiento de un equipo y he } \\
\text { aportado propuestas para la } \\
\text { elaboración y aplicación de los } \\
\text { métodos y procedimientos de trabajo } \\
\text { cooperativo en el grupo. }\end{array}$ & & & & & & & & & & & & \\
\hline
\end{tabular}

Figura 3. Instrumento para la autoevaluación y coevaluación del trabajo en equipo Fuente: adaptado de Pastor del Pino et al. (2017).

En la fase 4 (seminario 5) se repitió la autoevaluación realizada en la fase 1 y la evaluación entre iguales de la fase 2 .

La fase 5 se realizó durante la última semana de clase. Se llevó a cabo la exposición y defensa del proyecto, otorgando el tribunal de profesores una calificación al resultado del proyecto trabajado en equipo. A partir de esta calificación del tribunal, los estudiantes otorgaron una nota individual para cada uno de los miembros integrantes del equipo.

Finalmente, respetándose el anonimato de todos los participantes, se realizó la entrevista semiestructurada en cada uno de los cursos respondiendo 28 estudiantes durante el curso 2016-2017 y 54 estudiantes durante el curso 2017-2018 (N=82).

\subsection{Análisis de datos}

Para el análisis de los datos se realizó en primer lugar un microanálisis, codificación abierta, codificación axial y codificación selectiva basados en la Teoría Fundamentada (Strauss y Corbin, 2002); el método comparativo constante fue la técnica utilizada para la codificación (Flick, 2004) con la ayuda del software de investigación cualitativa NVivo 8.0.

\section{Resultados}

Los resultados obtenidos del análisis cualitativo de los datos mostraron la emergencia de tres categorías principales en las percepciones de los estudiantes sobre la aplicación de una evaluación formativa en el trabajo en equipo. En primer lugar, se hallaron los significados que de manera general los estudiantes atribuyeron a los trabajos en equipo. 
En segundo lugar, se posicionaron con respecto a la experiencia vivida sobre la aplicación de una evaluación formativa para mejorar el trabajo en equipo. Y, finalmente, aludieron a determinadas creencias sobre el sistema de calificación mediante la negociación y el pacto entre los propios estudiantes que trabajaron en equipo.

\subsection{Significados que los estudiantes atribuyeron a los trabajos en equipo}

Cuatro significados fundamentales atribuyeron los estudiantes a los trabajos en equipo (figura 2). Dos de ellos fueron de tendencia positiva, uno, de tendencia negativa y, otro, interpretable según el contexto.

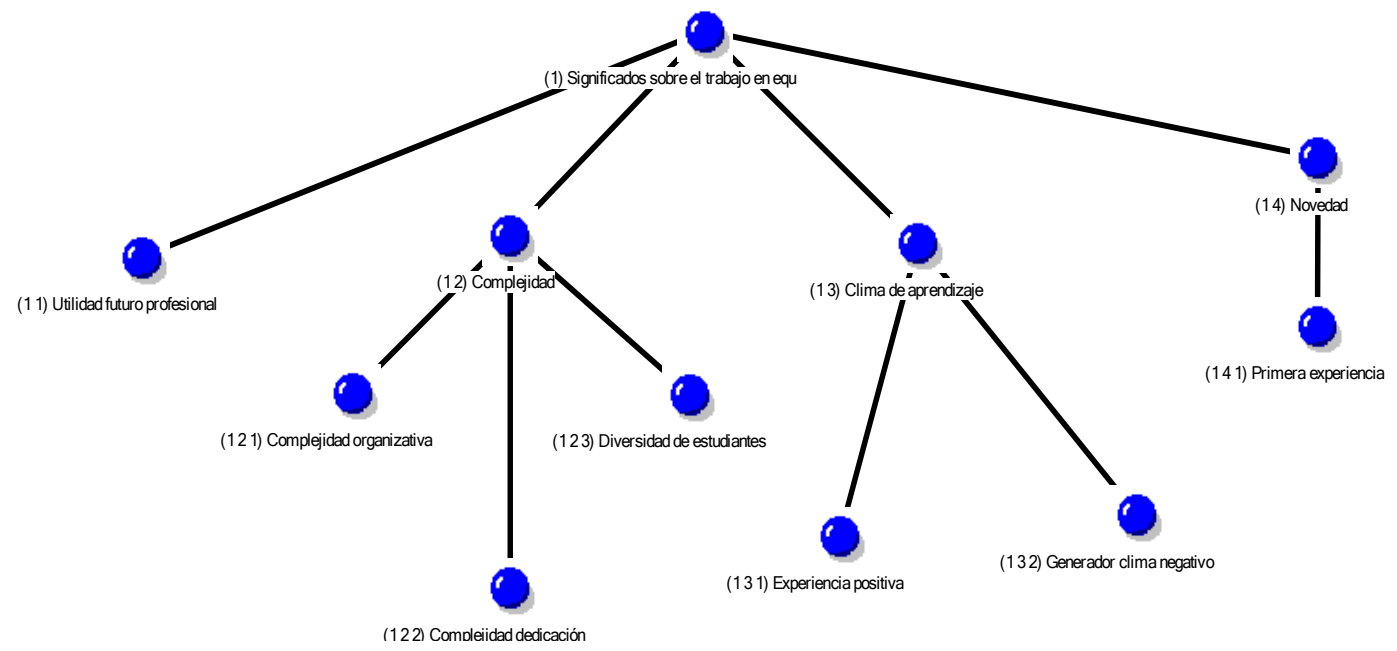

Figura 4. Significados que los estudiantes atribuyeron al trabajo en equipo Fuente: Elaboración propia.

Comenzando por los significados de tendencia positiva, resaltaremos que una parte de los estudiantes consideraron que la realización de trabajos en equipo es un acierto en la Educación Superior, reconociendo su utilidad para el futuro profesional inmediato.

Considero importante el saber trabajar en equipo pues en la mayoría de los trabajos es imprescindible. (Section O, Paragraph 142, 101 characters)

Igualmente, aunque de forma aislada, se encontraron estudiantes que no habían tenido experiencias previas en trabajos en equipo, por lo que el significado que le atribuyeron los estudiantes al mismo fue el de la novedad. En efecto, esto nos conduce a pensar que el trabajo en equipo en la educación superior no es una práctica tan extendida como en realidad nos parece. Bien es cierto, que esta novedad es entendida por los estudiantes como una referencia positiva; una referencia que parece conferir valor a la formación del estudiante.

El hecho de trabajar en equipo ha sido bastante nuevo para mí. (Section 0, Paragraph 7, 62 characters)

Por el contrario, las apreciaciones de los estudiantes centradas en significados de tendencia negativa fueron abundantes y se ciñeron a la complejidad. En su opinión, no resulta sencillo realizar trabajos en equipo, sino que más bien les parece complejo. Aludieron a la complejidad organizativa y la complejidad de la dedicación; así como, a la complejidad por la diversidad de perfiles de responsabilidad, personalidad y liderazgo. 
Creo que hay gente que toma exceso de responsabilidades cuando nadie se lo ha otorgado, también hay gente que le pone pegas a todo. (Section O, Paragraph 158, 188 characters)

$\mathrm{Y}$, finalmente, otro significado que otorgaron al trabajo en equipo fue el centrado en la idea del clima de aprendizaje. Mientras que los estudiantes, en bastantes casos, asocian el trabajo en equipo a experiencias con buen clima de aprendizaje, en algún caso, se defiende con rotundidad que el trabajo en equipo es generador de clima negativo y de tensión, nada favorecedor del aprendizaje.

...hace que los alumnos tengan que tragar gente y trabajo que no quieren, por lo que más que constructivo es destructivo, creándose tensiones y mal ambiente de trabajo durante 4 meses. (Section 0, Paragraph 57, 226 characters)

\subsection{La experiencia vivenciada con la aplicación de evaluación formativa para mejorar el trabajo en equipo}

Con respecto a la experiencia vivenciada se encontraron tres categorías principales: la importancia que los estudiantes le atribuyeron al rol del docente, la efectividad de la evaluación formativa en la evolución de la responsabilidad y la dificultad manifestada con respecto a la evaluación entre iguales (figura 5).

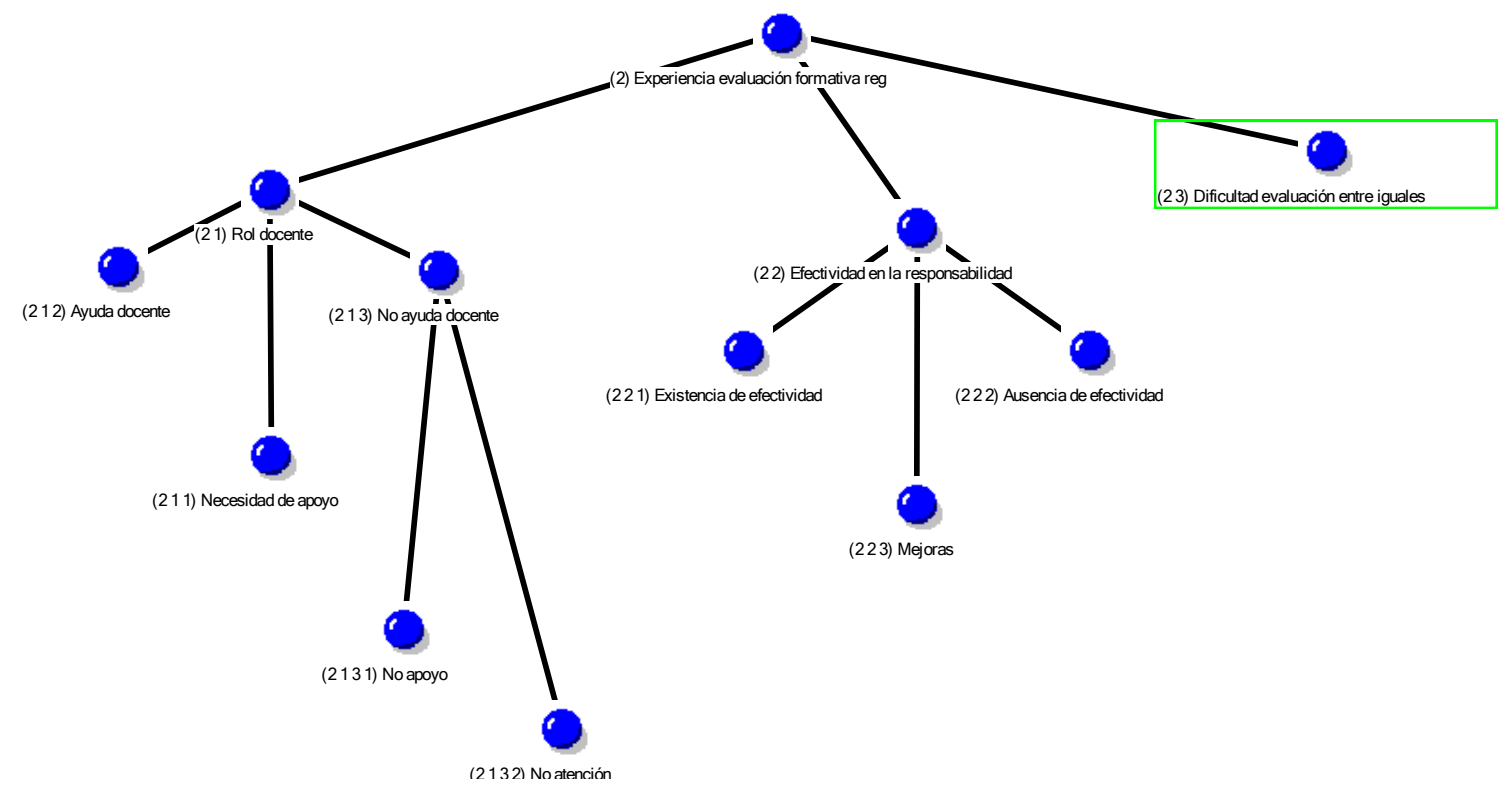

Figura 5. Experiencia vivenciada con la aplicación de una evaluación formativa para mejorar el trabajo en equipo Fuente: Elaboración propia.

La existencia de efectividad en la responsabilidad fue sin duda la aportación más firme de los estudiantes. Fueron innumerables las alusiones de los estudiantes a conceptos de interés formativo. Expusieron la idea de la eficacia en el aprendizaje, considerando que la experiencia aportaba aprendizaje organizativo, madurez en la asunción de compromisos, seguridad y saber hacer ante otras personas del grupo; igualmente, aludieron a la mejora de su formación en convivencia durante el trabajo en equipo, considerando haberse implicado en asuntos de resolución de conflictos, tolerancia, aceptación de ideas de otros y acuerdos; y, finalmente, aludieron a la mejora de la formación en cooperación, como el 
compromiso con los demás, la atención a las repercusiones, el sentirse parte importante del grupo y la importancia de la participación de todos para conseguir el éxito.

No obstante, también plantearon algunas posibles mejoras y expresaron que era necesario que los estudiantes tuviesen ciertos niveles previos de motivación y responsabilidad, ya que sin ello, la experiencia del trabajo en equipo con evaluación formativa no surtía efecto alguno en la responsabilidad de los estudiantes.

Destacamos como idea importante y, a considerar en futuros estudios, la exigencia que plantearon los estudiantes sobre la necesidad del apoyo del profesor. Lo entendieron como esencial y, curiosamente, las necesidades de los estudiantes ante este asunto fueron diferentes. Mientras algunos grupos parecieron conformarse con el apoyo realizado por el profesor, en otros casos, consideraron fundamental que este apoyo fuese más elevado; sobre todo, cuando los equipos padecieron conflictos serios. Merecería la pena insistir en el estudio del rol del profesor y sus funciones durante el desarrollo de los trabajos en equipo con implementación de evaluación formativa, dado que el requerimiento diferenciado por parte de los estudiantes, queda sin duda patente en este estudio.

...no digo que no sea correcto, pero propongo más apoyo en la gestión del grupo por parte del professorado. (Section O, Paragraph 68, 103 characters.)

No fomentan el trabajo en grupo y se lavan las manos cuando un grupo tiene problemas. (Section 0, Paragraph 16, 85 characters.)

Finalmente, se destacó la idea de que la evaluación formativa había ayudado a la efectividad para la mejora de responsabilidad, pero la idea de utilizar un instrumento de evaluación entre iguales como instrumento de evaluación formativa para mejorar el trabajo en equipo no estuvo exento de dificultad para los estudiantes. Los estudiantes señalaron dificultades afectivas cuando debieron posicionarse, valorando positiva o negativamente la conducta de sus compañeros, durante la realización del trabajo en equipo; por ello, expresaron preferir los instrumentos de autoevaluación.

...Con la coevaluación no me gusta poner nota a mis amigos, me cuesta separar lo afectivo, a mí me gustaría que las personas fuésemos honestas y reconociésemos lo que ha hecho cada uno. (Section O, Paragraph 32, 185 characters)

\section{Creencias de los estudiantes sobre el sistema de calificación pactado en equipo}

Con respecto a las creencias de los estudiantes sobre el sistema de calificación elegido y pactado previamente por el equipo (figura 4) quedó claro el posicionamiento mayoritario de los estudiantes. Consideraron que resultó útil para la responsabilidad y la justicia en la calificación,

Útil si, ya que cada uno obtendrá la nota que merece en función del trabajo que haya realizado. (Section 0, Paragraph 202, 32 characters)

Pero, no fueron pocos los que señalaron problemas de desigualdad en el esfuerzo por parte de los estudiantes y la dificultad extrema de plasmarlo en una calificación; así como, los que expresaron sensaciones de injusticia en la calificación por las diferentes percepciones de esfuerzo referidas a uno mismo y a los demás. Destacar también la idea aislada pero curiosa de un estudiante, que percibió incoherencia en recibir una calificación individual tras haber realizado un trabajo en equipo en el que se presupone un objetivo y un resultado común; y, por último, alguna apreciación de mal ambiente o sensación negativa que el 
reparto de la calificación generó entre los miembros del propio grupo, por diferencias de percepción en la participación.

Sí, pero la gente que menos ha participado, va a seguir calificándose de más, lo que seguirá afectando positivamente a su nota. (Section O, Paragraph 192, 81 characters)

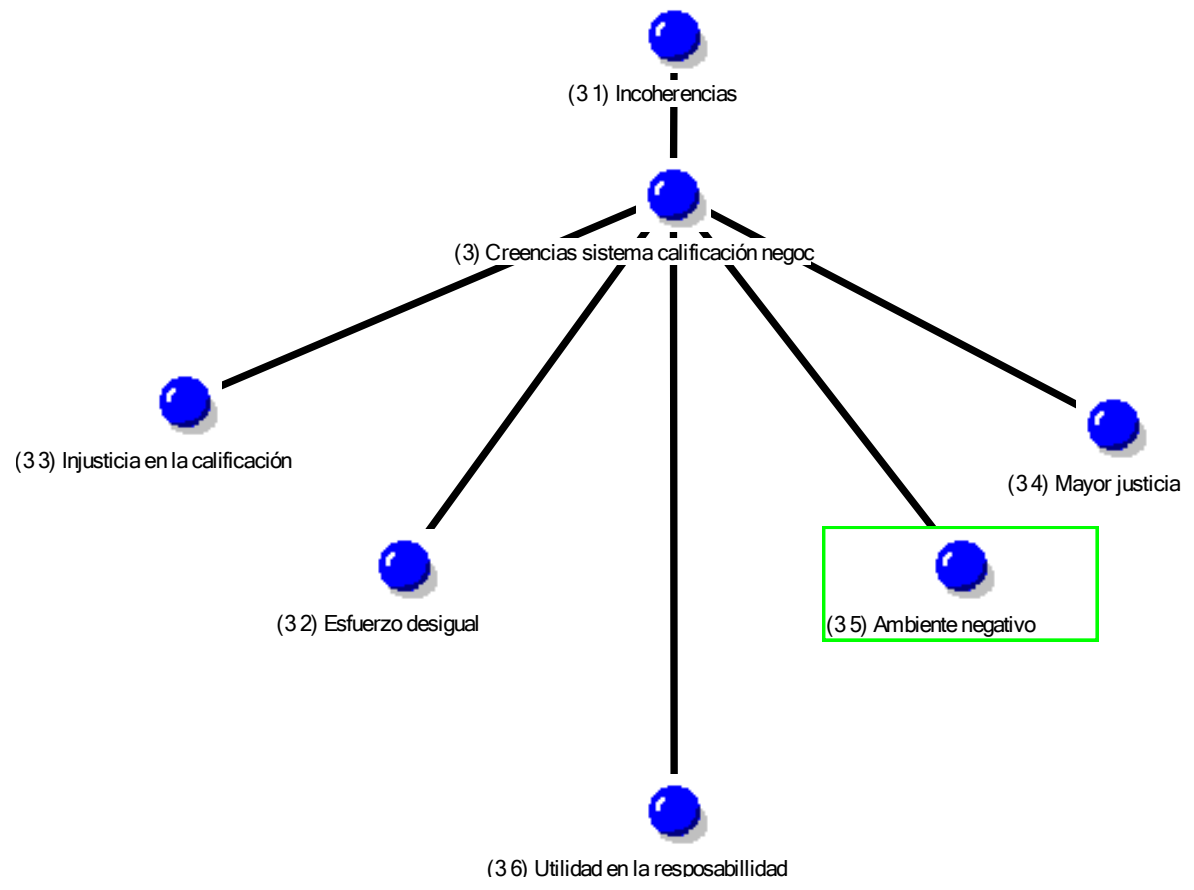

Figura 4. Creencias de los estudiantes sobre el sistema de evaluación pactado en equipo Fuente: Elaboración propia.

\section{Discusión y conclusiones}

El aprendizaje basado en competencias constituye hoy en día el núcleo central de la programación docente en Educación Superior. Sin embargo, a pesar de las directrices europeas sobre la importancia de la formación integral del individuo recogidas en la Declaración de Lisboa (Parlamento Europeo, 2000), todavía se siguen desarrollando más las competencias que definen el perfil profesional que las genéricas (Chica, 2011). En este sentido, para nuestro alumnado, la experiencia realizada para desarrollar la competencia de trabajo en equipo, considerada competencia transversal o genérica, ha supuesto una novedad. Siendo que se encuentran en el tercer curso del Grado, es un aspecto que hay que valorar y reflexionar, ya que si se considera necesario aprender a trabajar en equipo, tal vez sea interesante empezar a desarrollar esta competencia desde los primeros cursos.

La individualidad laboral ha quedado relegada a un segundo plano frente al trabajo en equipo. Según Hollenbeck et. al., (2004) desde los años 80 comenzó a tener importancia esta manera de trabajar, extendiéndose rápidamente a partir de los años noventa (Park et. al. 2005). Nuestros estudiantes han mostrado estar sensibilizados al respecto ya que han valorado la importancia de aprender a trabajar en equipo para su futuro laboral, aunque, inciden en la complejidad a la hora de realizar el trabajo propuesto debido a dificultades 
encontradas en cuanto a la organización, las horas requeridas de dedicación y la diversidad de perfiles entre los compañeros.

Respecto a la complejidad, la opinión de los estudiantes coincide con lo señalado por Cardona y Wilkinson (2006), los cuales indican que para que el trabajo sea eficaz son necesarias una serie de capacidades para saber organizarse y gestionar el tiempo, aceptar la opinión de los demás, proponer ideas, adaptarse, etc. Los conflictos más comunes que surgen entre el alumnado al realizar un trabajo en equipo fueron analizados por Del Canto et al. (2009), habiendo encontrado como principal problema el nivel de implicación de cada miembro del equipo. No todos los estudiantes se esfuerzan de la misma manera, sin embargo, en general, todos obtienen la misma calificación. En este sentido, Cuadrado et al. (2012) señalan que cuando la calificación es la misma para todos se corre el riesgo de que algún alumno no se implique en la tarea y se beneficie del trabajo de los demás. En esta línea, varios estudios han intentado introducir la autoevaluación y la coevaluación como herramientas para el reparto de la calificación individual en el trabajo grupal, aportando diferentes sistemas para facilitar el reparto de la nota (Goldfinch y Raeside, 1990, Jiménez 2006, Morales, 2008).

En nuestro estudio, el profesorado decidió que la calificación individual fuese pactada entre los miembros del equipo y para ello se mostró a los estudiantes dos sistemas para el reparto de la calificación propuestos por Morales (2008). Al finalizar la experiencia, los alumnos indicaron la utilidad de haber elaborado previamente y bajo consenso el sistema de reparto de la calificación, utilizando rúbricas o escalas elaboradas por cada equipo. Por tanto, el trabajo realizado en los seminarios para desarrollar la responsabilidad personal y social en el trabajo grupal, y la sensibilización sobre su repercusión en la calificación final, fue positivo y enriquecedor. Sin embargo, el cambio de la evaluación del equipo con la evaluación individual de sus miembros, tal y como proponían Cuadrado et al. (2013), para solventar el problema citado anteriormente respecto a los alumnos que no se implican en la tarea, no se zanjó totalmente. Algunos alumnos continuaron mostrando su descontento respecto a la desigualdad en el esfuerzo, e indicaron que de todas formas, aquellos alumnos con un bajo nivel de implicación iban a conseguir una calificación superior a la que se merecían, debido a su falta de responsabilidad en el momento de autoevaluarse.

Tal y como advertían Canto et al. (2009), el nivel del motivación y ambición de los estudiantes es diferente, por lo que es necesario trabajar la responsabilidad personal y social en el trabajo en equipo, para que cada alumno sea capaz de aceptar el trabajo realizado, y sea consecuente y justo consigo mismo y con los demás. Siendo esto último fundamental, ya que un mal estudiante podrá librarse de trabajar en un trabajo grupal pero, posteriormente, cuando se incorpore al mundo laboral, si no cumple con sus responsabilidades sufrirá serias consecuencias (Kaufman, Felder y Fuller, 2000). Nuestros resultados señalan una mejora reconocida en el aprendizaje, la implicación y la justicia, si bien, se siguen detectando algunos inconvenientes en la percepción y valoración del esfuerzo y la participación ajenos.

Algunos autores definen el trabajo en equipo como "la pedagogía de la democracia" permitiendo la preparación del estudiante como ciudadano (Cuadrado et al, 2012). Y es que entre otras cosas, en la vida hay que tomar decisiones y ser capaz de discernir entre lo personal y lo profesional. En este sentido, a los estudiantes les ha resultado complicado dejar de lado lo personal para evaluar el trabajo realizado por cada uno de sus compañeros. 
Aunque en alguno de los casos no hayan sido capaces de ser justos debido a la amistad, el simple hecho de haber tenido que reflexionar y valorar, quizás les haya preparado para en un futuro ser capaces de asumir la responsabilidad personal que les sea requerida.

Por otro lado, ha surgido un interesante debate respecto al papel del profesorado en el trabajo en equipo. Algunos estudiantes muestran la necesidad de una mayor implicación del profesorado para poder resolver posibles problemas y conflictos. ¿El docente ha de ser el guía o tiene que intervenir? Hemos visto que no es sencillo trabajar en equipo. Los alumnos señalaban la dificultad de tener que realizar un trabajo con compañeros con diferentes inquietudes y con los que quizás no querían colaborar. Aunque hay que tener presente que uno de los principales objetivos es desarrollar la responsabilidad personal y social entre los miembros del equipo, y que para ello tienen que ser ellos los que aprendan a trabajar de este modo, organizarse y regularse, utilizando para ello las herramientas aportadas por el docente, tal vez en determinadas ocasiones y en función del grupo, sea necesario que el profesor intervenga para ayudarles a resolver conflictos.

En base a todo lo comentado anteriormente y a los resultados obtenidos, señalar que nos encontramos ante una experiencia compleja, de aspectos controvertidos y que atañe a relaciones esencialmente humanas como son las basadas en habilidades interpersonales e intrapersonales de muy difícil captación y manejo. La competencia de trabajo en equipo engloba a la persona en todas sus dimensiones, como ser autónomo y social, y ubica a los estudiantes en un proceso formativo verdaderamente enriquecedor.

En conclusión, destacar que el desarrollo de la competencia genérica relacionada con el trabajo en equipo fue reconocido por los estudiantes como útil para su futuro laboral.

Igualmente, a pesar de la complejidad percibida por los universitarios en el trabajo de equipo realizado durante la "buena práctica", la satisfacción y el reconocimiento del aprendizaje, la convivencia y la cooperación en procesos tan importantes como la organización, la toma de acuerdos, la resolución de conflictos, la responsabilidad, la asunción de consecuencias y la maduración personal fueron, sin lugar a dudas, cuestiones altamente formativas para los estudiantes, y efectivamente, esenciales en la formación de un buen profesional durante el periodo universitario.

Los estudiantes percibieron bastante justicia en la calificación del trabajo en equipo por la implementación del reparto de la calificación con un sistema previo negociado y pactado por los propios miembros del equipo, si bien, se vislumbró un atisbo de incoherencia por la individualización de la calificación en trabajos en los que se presuponen objetivos cooperativos, solidarios y comunes.

Finalmente, señalar la limitación de los hallazgos de este estudio, al ser contextualizado en un grupo concreto y una universidad específica, no teniendo por ello la intención de generalizar ni de inferir dichos resultados a otras poblaciones o contextos. Como prospectiva para próximas experiencias, exponemos la necesidad de ahondar en la eficacia o ineficacia de los procesos de evaluación entre iguales, cuando entra en juego una posible calificación; asimismo, la necesidad de estudiar y determinar el rol del profesor en este tipo de trabajos, como podría ser, el de identificador de niveles de desarrollo de la competencia del trabajo en equipo, así como, el de orientador en el progreso de este desarrollo en los estudiantes universitarios, y mediador en los conflictos difíciles de resolver por los propios estudiantes. 


\section{Agradecimientos}

Agradecimientos a la Red Nacional de Evaluación Formativa y Compartida por su apoyo a la fundamentación de la Buena Práctica y a la Universidad de Zaragoza por su financiación mediante la convocatoria de Proyectos de Innovación Docente (PIDUZ_17_396).

\section{Referencias}

Aguirre-García, J. C. y Jaramillo-Echeverri, L. G. (2012). Aportes del método fenomenológico a la investigación educativa. Revista Latinoamericana de Estudios Educativos, 8(2), 51-74.

Bain, K. (2005). Lo que hacen los mejores profesores universitarios. Valencia: Universidad de Valencia.

Boud, D. y Falchikov, N. (2007). Rethinking assessment in higher education .Learning for the long term. Oxon: Routledge.

Brockbank, A. y McGill, I. (2006). Facilitating Reflective Learning through Mentoring and Coaching. Londres: Kogan Page.

Brown, S. y Glasner, A. (1999). Assessment matters in higher education. Buckingham: Open University Press.

Cabero, J. y Barroso, J. (2013). La utilización del juicio de experto para la evaluación de TIC: el coeficiente de competencia experta. Bordón, 65(2), 25-38.

https://doi.org/10.13042/brp.2013.65202

Cardona, P. y Wilkinson, H. (2006). Trabajo en equipo. Occasional Paper, o7(10), 1-10.

Carless, D. (2006). Learning-oriented assessment: principles and practice. Assessment \& Evaluation in Higher Education, 31 (4), 395-398. https://doi.org/10.1080/02602930600679043

Chica, E. (2011). Una propuesta de evaluación para el trabajo en grupo mediante rúbrica. Escuela Abierta, 14, 67-81.

Creswell, J. W. (2011). Educational research. Planning, conducting and evaluating quantitative and qualitative research. Boston, MA: Pearson.

Clemente, J. S. y Escribá C. (2013). Análisis de la percepción de las competencias genéricas adquiridas en la Universidad. Revista de Educación, 362, 535-561. https://doi.org/10.4438/1988-592X-RE-2013-362-241

Cuadrado, C., Fernández-López, F., Fernández-López, M., Fernández-Pacheco, C. González, D. y Lifante, I. (2012). Técnicas de trabajo en equipo para estudiantes universitarios. X Jornadas de redes de investigación en docencia universitaria. Alicante: Universidad de Alicante.

De Miguel, M. (2005). Modalidades de enseñanza centradas en el desarrollo de competencias. Orientaciones para promover el cambio metodológico en el EEES. Madrid: MEC/Universidad de Oviedo.

Del Canto, P., Gallego, I., López, J. M., Mora, J., Reyes, A., Rodríguez, E., Sanjeevan, K., Santamaría, E. y Valero, M. (2009). Conflictos en el trabajo en grupo: cuatro casos habituales. Revista de Formación e Innovación Educativa Universitaria, 2(4), 211-226.

Denton, D. (2014). Using Screen Capture Feedback to Improve Academic Performance.TechTrends. Linking Research and Practice to Improve Learning, 58(6), 51-56. https://doi.org/10.1007/s11528-014-0803-0. 
Domingo, J. (2008). El aprendizaje cooperativo. Cuadernos de Trabajo Social, 21, 231-246.

Flick, U. (1998). An introduction to qualitative research. Londres: Sage Publications.

Goldfinch, J. y Raeside R. (1990). Development of a peer assessment technique for obtaining individual marks on a group project. Assessment $\Xi^{2}$ Evaluation in Higher Education, 15(3), $210-$ 231. https://doi.org/10.1080/0260293900150304

González Morga, N. (2018). Las competencias transversales en la universidad: Propiedades psicométricas de un cuestionario. Educacion XX1, 21(1), 231-262. https://doi.org/ 10.5944/educXX1.15662

González, J. y Wagenaar, R. (2003). Tuning educational structures in Europe. Bilbao: Universidad de Deusto.

Hollenbeck, J. R., DeRue, D. S. y Guzzo, R. (2004). Bridging the gap between I/O research and HR practice: Improving team composition, team training, and team task design. Human Resource Management, 43(4), 353-366. https://doi.org/10.1002/hrm.20029

Hortigüela, D., Pérez-Pueyo, Á. y Abella, V. (2015). ¿Cómo influye el sistema de evaluación en la percepción del alumnado? @TIC revista d’innovació educativa, 14, 82-89.

https://doi.org/10.7203/attic.14.4170

Howe, R. (2011). Assessing Team work. College documentation bulletin, 7. Recuperado de http://regard.cdc.qc.ca/CDC/Pages/Front/Accueil/Accueil.aspx\#lisucc

Ibarra, M. S. y Rodríguez-Gómez, G. (2010). Aproximación al discurso dominante sobre la evaluación del aprendizaje en la universidad. Revista de Educación, 351, 385-407. Recuperado de http://www.revistaeducacion.educacion.es/re351/re351_16.pdf

Jiménez Valverde, G. (2006). Obtención de notas individuales a partir de una nota de grupo mediante una evaluación cooperativa. Revista Iberoamericana de Educación, 38(5), 1-15.

Kaufman, D. B.; Felder, R. M. y Fuller, H. (2000). Accounting for individual efforts in cooperative learning teams. Journal of Engineering Education, 89(2), 133-140. https://doi.org/10.1002/j.2168-9830.2000.tb00507.x

Lizandra, J., Valencia-Peris, A., Aienza, R. y Martos D. (2017). Itinerarios de evaluación y su relación en el rendimiento académico. REDU: Revista de Docencia Universitaria, 15(2), 315-328. https://doi.org/10.4995/redu.2017.7862

López-Pastor, V. M., Pintor, P., Muros, B. y Webb, G. (2013). Formative Assessment Strategies and their Effect on Student Performance and on Student and Tutor Workload: The Results of Research Projects Undertaken in Preparation for Greater Convergence of Universities in Spain within the European Higher Education Area (EHEA). Journal of Further and Higher Education, 37(2), 163-180. https://doi.org/10.1080/0309877X.2011.644780

Martínez-Clares, P. y González-Morga, N. (2018). Las competencias transversales en la universidad: propiedades psicométricas de un cuestionario. Educación XX1, 21(1), 231-262.

Michavila, F., Martínez, J. M., Martín- González, M., García-Peñalvo, F. J. y Cruz-Benito, J. (2016). Barómetro de empleabilidad y empleo de los universitarios en España, 2015 (Primer informe de resultados). Madrid: Observatorio de Empleabilidad y Empleo Universitario.

Morales, P. (2008). Estrategias para evaluar y calificar el producto del equipo: cómo diferenciar las calificaciones individuales. En L. Prieto (Coord.), La enseñanza centrada en el aprendizaje: estrategias útiles para el profesorado (pp.151-169). Barcelona: Octaedro.

Parlamento Europeo (2000). Consejo Europeo Lisboa 23 y 24 de Marzo 2000: Conclusiones de la Presidencia. Recuperado de: http://www.europarl.europa.eu/summits/lis 1_es.htm 
Pastor del Pino, M. C. et al. (2017). Guía para la integración en la docencia de la competencia 2: trabajar en equipo. Recuperado de http://repositorio.upct.es/bitstream/handle/10317/4100/gid2.pdf;jsessionid=57027123 D8722E071990F7F2612D1877? sequence=1

Patton, M. Q. (1990). Qualitative evaluation and Research Methods. Londres: Sage.

Pérez-Pueyo, A., Hortigüela, D. y Hernando, A. (2013). La coevaluación intragrupal y el reparto de notas bajo un proceso de evaluación formativa. En P. Membiela, N. Casado y M. I. Cebreiros, Experiencias e innovación docente en el contexto actual de la docencia universitaria (pp. 285-289). Orense: Educación Editora.

Prieto, J. R, Alarcón, D. y Fernández, C. B. (2018). Aprendizaje y evaluación de competencias en el universitario de ciencias sociales. REDU: Revista de Docencia Universitaria, 16(1), 193-210. https://doi.org/10.4995/redu.2018.8941

Proulx, J. (2009). Enseigner: réalité, réflexions et pratiques. Trois-Rivières: Cégep de Trois-Rivières.

Romero-Martín, R., Fraile-Aranda, A., López-Pastor, V. M. y Castejón-Oliva, F. J. (2014). Relación entre sistemas de evaluación formativa, rendimiento académico y carga de trabajo del profesor y del alumno en la docencia universitaria. Infancia y Aprendizaje, 37(1),16-32. https://doi.org/10.1080/02103702.2014.9188118

Recomendación del Parlamento Europeo y del Consejo (2006). Las competencias clave para el aprendizaje permanente. Recuperado de http://eurlex.europa.eu/LexUriServ/LexUriServ.do?uri=OJ:L:2006:394:0010:0018:ES:P DF

Park, S., Henkin, A. B. y Egley, R. (2005). Teacher team commitment, teamwork and trust: Exploring associations. Journal of Educational Administration, 43(5), 462-479. https://doi.org/10.1108/09578230510615233

Sánchez-Elvira, A., López-González, M. A. y Fernández-Sánchez, M. V. (2010). Análisis de las competencias genéricas en los nuevos títulos de grado del EEES en las universidades españolas. REDU: Revista de Docencia Universitaria, 8(1), 12-35.

Scallon, G. (2004). L'évaluation des apprentissages dans une approche par compétences. Saint-Laurent: Éditions du Renouveau Pédagogique.

Strauss, A. y Corbin, J. (2002). Bases de la investigación cualitativa. Técnicas y procedimientos para desarrollar la teoría fundamentada. Medellín: Universidad de Antioquía.

Utha, K. (2015). Formative assessment practices in Buthanese Secondary Schools and its impact on qualiy of education. Tesis doctoral. Aalborg University Denemark.

Villa, A. y Poblete, M. (2007). Evaluación de competencias genéricas: Principios, oportunidades y limitaciones. Bordón, 63(1), 147-170.

Zabalza, M. Á. (2012). Las competencias en la formación del profesorado: de la teoría a las propuestas prácticas Tendencias pedagógicas, 20, 5-32.

\section{Cv de los autores}

\section{Sonia Asún Dieste}

Doctora en Ciencias de la Actividad Física y del Deporte por la Universidad de Zaragoza. Profesora Titular en la Facultad de Ciencias de la Salud y del Deporte de la Universidad 
de Zaragoza. Las líneas de investigación se centran en la evaluación formativa en educación superior y educación secundaria y en la educación física. Participación en proyectos de investigación, desarrollo e innovación a nivel nacional y aportaciones científicas diversas en revistas y Congresos Nacionales e Internacionales. Miembro de la Red Nacional de Evaluación Formativa y Compartida en Educación Superior. Miembro de la Agencia Estatal de Investigación Científica. ORCID ID: 0000-0002-3761-2903. Email.sonasun@unizar.es

\section{Marta Rapún López}

Doctora en Ciencias de la Actividad Física y del Deporte por la Universidad de Zaragoza. Profesora de Educación Secundaria en la especialidad de Educación Física. Profesora Asociada en la Facultad de Ciencias de la Salud y del Deporte de la Universidad de Zaragoza. Las líneas de investigación se centran en la evaluación formativa en educación superior y educación secundaria y en la educación física. Participación en proyectos de investigación e innovación a nivel nacional y aportaciones científicas diversas en revistas y Congresos Nacionales e Internacionales. Miembro de la Red Nacional de Evaluación Formativa y Compartida en Educación Superior. Email.mrapun@unizar.es

\section{$M^{a}$ Rosario Romero Martín}

Doctora en Ciencias de la Actividad Física y del Deporte por la Universidad de Zaragoza. Profesora Titular en la Facultad de Ciencias de la Salud y del Deporte de la Universidad de Zaragoza. Líneas de investigación: enseñanza de la actividad física, expresión corporal y emociones en la actividad física. Miembro de la Red Nacional de Evaluación Formativa y Compartida en Educación Superior (REFYCE). Miembro de AFYEC (Asociación de Profesores de Actividad Física y Expresión Corporal). ORCID ID: 0000-0002-5019-4574. Email: rromero@unizar.es 